EDESIO MARTINS

RUFFO FREITAS-JUNIOR ${ }^{2}$

Maria Paula Curado ${ }^{3}$

Niliceana Maya Aires Frettas ${ }^{4}$

José CARLos de OliverRA ${ }^{5}$

Carleane Maciel Bandeira e Sillya ${ }^{6}$
Artigo original

Palavras-chave

Câncer de mama/diagnóstico Câncer de mama/epidemiologia Carcinoma in situ/epidemiologia Estadiamento de neoplasias

Keywords

Breast neoplasms/diagnosis Breast neoplasms/epidemiology Carcinoma in situ/epidemiology Neoplasm staging

\section{Evolução temporal dos estádios do câncer de mama ao diagnóstico em um registro de base populacional no Brasil Central}

\author{
Temporal evolution of breast cancer stages in a population-based cancer \\ registry in the Brazilian Central region
}

\section{Resumo}

OBJETIVO: Analisar a mudança temporal do estádio clínico do câncer de mama ao diagnóstico em mulheres residentes em Goiânia entre 1989 e 2003. MÉTODOS: estudo retrospectivo, utilizando-se a base de dados do Registro de Câncer de Base Populacional de Goiânia. As variáveis estudadas foram: idade, diagnóstico histológico, localização do tumor, tipo histológico e estádio clínico da doença. O período estudado foi dividido em três quinquênios: de 1989 a 1993, de 1994 a 1998 e de 1999 a 2003. Utilizou-se o teste Z para comparação das frequências da extensão ao diagnóstico por quinquênio. RESULTADOS: foram identificados 3.204 casos de câncer de mama. A média de idade foi de 56 anos (dp \pm 16 anos). Quanto ao estádio da doença, evidenciou-se que 45,6\% dos casos eram localizados na mama, com aumento de 19,2\% entre o primeiro e o terceiro quinquênio ( $p<0,001$; IC95\%=0, 14-0,23) e 10,2\% de casos eram metastáticos, Entretanto, foi observada uma redução de 17,7\% para os casos metastáticos no mesmo período ( $p<0,001 ; \mathrm{IC} 95 \%=0,14-0,21)$. A taxa de casos in situ entre 1989 e 1993 foi de 0,2\%, aumentando para 6,2\% em 1999-2003 (p<0,001; IC95\%=4,9-7,4). CONCLUSÃO: observou-se um aumento dos casos de carcinoma in situ e de carcinomas invasores localizados somente na mama em detrimento de uma redução dos casos com metástases linfonodais e à distância.

\section{Abstract}

PURPOSE: To analyze the temporal changes of breast cancer staging at diagnosis among women living in Goiânia, Goiás, Brazil, between 1989 and 2003. METHODS: Retrospective and descriptive study in which the cases were identified from the Population-Based Cancer Registry of Goiânia for the period from 1989 to 2003. The variables studied were age, diagnostic method, topographic sublocation, morphology and breast cancer staging. Frequency analyses were carried out on the variables and means, and the medians for the age were determined. The SPSS ${ }^{\circledR} 15.0$ software was used for statistical analyses. RESULTS: A total of 3,204 breast cancer cases were collected. The mean age was 56 years ( $s d \pm 16$ years). With regard to clinical staging, $45.6 \%$ of the cases were found to be localized in the breast, with an increased rate of 19.25\% between the first and the third five-year period $(\mathrm{p}<0.001 ; \mathrm{Cl} 95 \%=0.14$ 0.23 and $10.2 \%$ of cases were with distant metastases. However, a reduction of $17.74 \%$ for metastatic cases in the same interval $(p<0.001$ e Cl $95 \%=0.14-21)$ was observed. The in situ case rate was $0.2 \%$ in $1989-1993$ and increased to $6.2 \%$ in $1999-2003$ ( $p<0.001$, IC95\%=4.9-7.4). CONCLUSION: The diagnostic profile of breast cancer in the city of Goiânia is changing. Substantial increases in the number of early breast cancer cases are being found in relation to the number of advanced cases.
Trabalho parcialmente custeado pela Fundação de Amparo à Pesquisa do Estado de Goiás (FAPEG), processo: 200710267000252. ' Epidemiologista do Registro de Câncer de Base Populacional de Goiânia da Associação de Combate ao Câncer em Goiás - ACCG Goiânia (GO), Brasil.

2 Professor adjunto do Departamento de Ginecologia e Obstetrícia da Universidade Federal de Goiás - UFG; médico titular do Serviço de Ginecologia e Mama do Hospital Araújo Jorge da Associação de Combate ao Câncer em Goiás - ACCG - Goiânia (GO), Brasil.

${ }^{3}$ Chefe do Departamento de Produção em Epidemiologia Descritiva da International Agency for Research on Cancer - IARC - Lyon, França. "Médica do Serviço de Radioterapia do Hospital Araújo Jorge da Associação de Combate ao Câncer em Goiás - ACCG - Goiânia (GO), Brasil

${ }^{5}$ Coordenador do Registro de Câncer de Base Populacional de Goiânia da Associação de Combate ao Câncer em Goiás - ACCG Goiânia (GO), Brasil.

${ }^{\circ}$ Codificadora do Registro de Câncer de Base Populacional de Goiânia da Associação de Combate ao Câncer em Goiás - ACCG Goiânia (GO), Brasil. 


\section{Introdução}

O câncer de mama é a neoplasia mais comum entre as mulheres, responsável por cerca de $20 \%$ de todos os tipos de câncer no mundo ${ }^{1}$. A incidência mundial vem crescendo substancialmente nesses últimos anos, passando de 572 mil casos, em 1980, para 1,15 milhão, em 2002. As maiores incidências são observadas em países desenvolvidos, como os da América do Norte (99,4/100.000), oeste da Europa e Austrália/Nova Zelândia, ambos com $84,6 / 100.000^{1}$. No Brasil, esperam-se 49.400 novos casos de câncer de mama para o ano de 2008, com um risco estimado de 50,7 casos para cada 100 mil mulheres ${ }^{2}$.

A neoplasia mamária representa a primeira causa de óbito por câncer entre as mulheres, e os coeficientes de mortalidade padronizados por idade mostraram uma tendência ascendente entre 1979 e 1999 3,4. Em Goiânia, a taxa padronizada de mortalidade pela população mundial de Segi foi de 14,87/100.000 em 1988, elevando-se para $18,1 / 100.000$ mulheres em $2002^{5}$.

Apesar de estar bem estabelecido que o diagnóstico precoce e o tratamento adequado interferem nas taxas de mortalidade e na prevalência da neoplasia ${ }^{6}$, poucos são os dados disponíveis quanto à extensão do tumor ao diagnóstico do câncer de mama no Brasil. Estudos realizados no Brasil há mais de dez anos evidenciavam que de 60 a $70 \%$ dos casos de câncer de mama eram detectados em estádios avançados ${ }^{7,8}$. Mais recentemente, alguns estudos mostraram uma tendência ao aumento do número de casos iniciais ao diagnóstico, porém foram séries pequenas ${ }^{9} \mathrm{ou}$ com base em dados de registros hospitalares ${ }^{10}$.

A ausência de informações dificulta a avaliação dos programas destinados ao diagnóstico precoce da neoplasia maligna da mama. Dessa forma, a proposta deste artigo foi avaliar a evolução temporal do estadiamento do câncer de mama ao diagnóstico em mulheres na cidade de Goiânia no período de 1989 a 2003.

\section{Métodos}

Trata-se de um estudo descritivo e retrospectivo que tomou por base de dados o Registro de Câncer de Base Populacional de Goiânia (RCBP-Goiânia) no período de 1989 a 2003. O estudo foi aprovado pelo Comitê de Ética em Pesquisa do Hospital Araújo Jorge da Associação de Combate ao Câncer em Goiás.

Os casos de câncer de mama feminina identificados na base de dados do RCBP-Goiânia foram aqueles diagnosticados entre 1989 e 2003 . O critério de inclusão dos seguiu a metodologia do RCBP-Goiânia, conforme previamente descrito em detalhes 5 e aqui relatado resumidamente. $\mathrm{O}$ RCBP-Goiânia coleta todos os casos diagnosticados como câncer e de pacientes que tenham residência estabelecida no município de Goiânia há pelo menos seis meses antes da data do diagnóstico. Essa coleta é feita em laboratórios que realizam exames anatomopatológicos, hospitais e clínicas especializadas no diagnóstico e tratamento de pacientes oncológicos em Goiânia. Os dados são coletados em formulário próprio, complementados e digitados em um banco de dados para serem analisados. A qualidade da informação segue todas as recomendações da International Agency for Research on Cancer (IARC) ${ }^{11}$.

Mulheres que tiveram o diagnóstico de câncer antes do estabelecimento de residência em Goiânia foram excluídas deste estudo.

As variáveis analisadas foram: a idade da paciente ao diagnóstico, localização do tumor, tipo histológico e estadiamento da doença.

Apesar de o estadiamento TNM preconizado pela União Internacional Contra o Câncer (UICC) ser o mais utilizado mundialmente na condução do câncer de mama, para os Registros de Câncer de Base Populacional o estadiamento utilizado segue as recomendações do IARC, com base na extensão da doença ${ }^{11}$, bem como pelo SEER nos Estados Unidos ${ }^{12}$.

Dessa forma, foi considerado estadiamento in situ quando o laudo histológico referia se tratar de carcinoma in situ, sem menção alguma de invasão. Foi considerado "localizado" o tumor invasor cujo exame histológico não apresentava comprometimento axilar e se a paciente não apresentasse metástases detectáveis por exame clínico ou de imagem. Foi considerado "locorregional" quando havia referência de comprometimento linfonodal descrito no exame histológico ou no exame clínico descrito pelo médico assistente. A categoria "comprometimento linfonodal” só foi iniciada pelo RCBP-Goiânia a partir de 1994, ou seja, antes dessa data, todos os casos de câncer de mama com comprometimento linfonodal eram classificados como metastáticos. Foi considerada "metástase à distância" quando havia a presença de tumor fora da mama e da axila homolateral confirmadas por exames de imagem e/ou exame cito-histológico.

Foram realizadas análises de frequência para todas as variáveis e análises de tendência central para determinação da média da idade.

Utilizou-se o teste $\mathrm{Z}$ para comparação das frequências do estadiamento ao diagnóstico por quinquênio. O software SPSS $^{\circledast}$ (Statistical Package for the Social Sciences) versão 15.0 foi utilizado para a confecção do banco de dados e análises estatísticas, e o editor de planilhas $\operatorname{Excel}^{\circledR} 2003$, para confecção das tabelas e gráficos.

\section{Resultados}

Foram coletados, pelo RCBP-Goiânia, 3.204 casos de câncer de mama no período de 1989 a 2003. A mediana 
verificada foi de 53 anos com desvio padrão (dp) de \pm 16 anos. Ainda em relação à idade, 15,2\% das mulheres tinham 40 anos ou menos, $27,8 \%$ entre 40 e 50 anos e $57 \%$ tinham mais de 50 anos de idade.

Foram registrados 644 casos de câncer de mama entre 1989 e 1993, 956 casos entre 1994 e 1998 e 1.604 no último período, entre 1999 e 2003 , observando-se um aumento de $249 \%$ entre o primeiro e o último quinquênio analisados. Do total de casos registrados, 94,7\% tiveram confirmação por exame histopatológico, $0,6 \%$ por exame citopatológico, 2,4\% pelo atestado de óbito e $2 \%$ dos casos foram identificados clinicamente e/ou por exame de imagem (Tabela 1).

O tipo histológico mais frequente foi o carcinoma ductal infiltrante, correspondendo a 84,6\% dos casos, seguido pelo carcinoma lobular ( $4,8 \%)$, e outras neoplasias corresponderam a $9,9 \%$ dos casos. Os sarcomas e os linfomas extranodais corresponderam a menos de $1 \%$ dos casos. Os casos de carcinoma ductal e lobular não invasivos corresponderam a 1,6\% de todos os casos.

Quanto ao estadiamento da doença, durante os 15 anos estudados, evidenciou-se que 45,6\% dos casos eram localizados, 19,7\% apresentavam tumores com extensão regional, 10,2\% tinham metástases à distância no momento do diagnóstico e 4,2\% eram tumores in situ. Nesta análise, 20,3\% dos casos não traziam informação quanto ao estadiamento da doença no momento do diagnóstico.

Observou-se que no primeiro quinquênio o percentual de casos in situ foi $0,2 \%$, aumentando para 6,2\% no último quinquênio ( $\mathrm{p}<0,001 ; \mathrm{IC} 95 \%=4,9-7,4)$. Já para os tumores invasores localizados apenas na mama, o percentual aumentou de 33,2 para $52,4 \%$, com um aumento de $19,2 \%$ entre o primeiro e o terceiro quinquênios, ( $\mathrm{p}<0,001$; e IC95\%=0,14-0,23). Contrariamente, foi observado que o percentual de casos de câncer diagnosticados como doença metastática sofreu redução: de $24,1 \%$ no primeiro, para $6,3 \%$ no último período, com diminuição de $17,7 \%$ ( $\mathrm{p}<0,001$; e IC95\%=0,14-0,21). Deve-se ressaltar que os casos de câncer de mama com comprometimento linfonodal eram classificados como metastáticos até o ano de 1994, mas com a melhoria e qualificação profissional dos registradores de câncer envolvidos na coleta e classificação dos casos, passaram a ser classificados como tumores regionais. No período aqui estudado, houve redução de 41,5 para $11,5 \%$ dos casos sem informação quanto ao estadiamento da doença (Figura 1).

\section{Discussão}

O presente estudo evidenciou que $57 \%$ das pacientes tinham mais de 50 anos de idade e 15,2\% tinham 40 anos ou menos. Esse dado corrobora com o relatado em outras
Tabela 1 - Frequências absolutas e relativas dos casos de câncer de mama segundo idade, base diagnóstica, extensão e período no município de Goiânia - 1989-2003 (n=3.204)

\begin{tabular}{|c|c|c|}
\hline Variável & n & $\%$ \\
\hline \multicolumn{3}{|l|}{ Idade (anos) } \\
\hline$\leq \mathbf{4 0}$ & 488 & 15,2 \\
\hline $41-50$ & 875 & 27,3 \\
\hline$>50$ & 1.841 & 57,5 \\
\hline \multicolumn{3}{|l|}{ Base de diagnóstico } \\
\hline Histopatológico & 3.035 & 94,7 \\
\hline Citológico & 30 & 0,9 \\
\hline Clínico & 48 & 1,5 \\
\hline Imagem & 13 & 0,5 \\
\hline Atestado de óbito & 78 & 2,4 \\
\hline \multicolumn{3}{|l|}{ Extensão } \\
\hline In situ & 133 & 4,2 \\
\hline Localizado & 1.461 & 45,6 \\
\hline Regional & 632 & 10,2 \\
\hline Metástase & 328 & 19,7 \\
\hline Sem informações & 650 & 20,3 \\
\hline \multicolumn{3}{|l|}{ Período } \\
\hline 1989-1993 & 644 & 20,1 \\
\hline 1994-1998 & 956 & 29,8 \\
\hline 1999-2003 & 1.604 & 50,1 \\
\hline
\end{tabular}

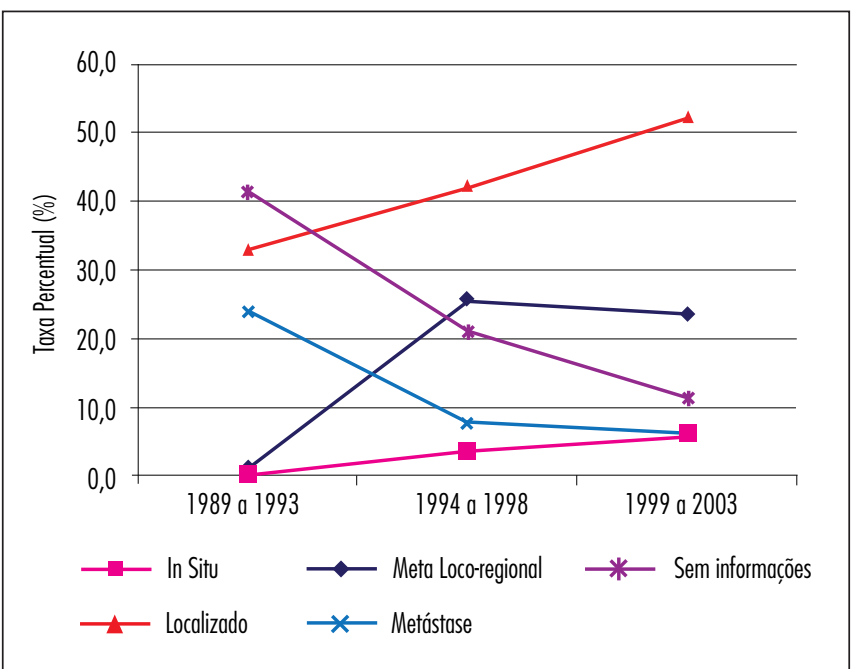

Figura 1 - Distribuição percentual dos casos de câncer de mama segundo o estadiamento da doença ao diagnóstico, por quinquênio, em Goiânia, 1989-2003.

séries de casos $^{13-16}$, o que confirma que o câncer de mama é mais frequente em mulheres com mais de 50 anos de idade $^{13,15}$. Em relação às mulheres jovens, o aumento da incidência do câncer de mama, observado por nós em estudo prévio, é inferior ao aumento nas faixas etárias acima de 50 anos $^{13}$, porém para as mulheres mais jovens, espera-se um pior prognóstico em comparação a mulheres acima dos 50 anos de idade ${ }^{14}$.

No presente estudo, evidenciou-se um aumento do número absoluto de casos de câncer da mama em $249 \%$ no período de 1999 a 2003. O principal motivo deste 
aumento esteve relacionado ao crescimento da população da cidade de Goiânia, mas ainda assim houve um aumento da incidência, principalmente na faixa etária de 50 a 59 anos, conforme publicado previamente ${ }^{13}$.

Até poucos anos atrás, um aumento do número de casos de câncer da mama vinha sendo relatado em todo o mundo, tanto nos países desenvolvidos ${ }^{1}$ quanto nos países em desenvolvimento ${ }^{13,16}$. Entretanto, após a redução do uso de terapia hormonal, em decorrência da publicação do estudo WHI, observou-se uma redução significativa da incidência do câncer de mama em alguns países $^{17,18 .}$

O fato de não ter havido diminuição da incidência da neoplasia mamária nos anos mais recentes pode ser explicado pela pequena redução do uso de terapia hormonal entre as mulheres brasileiras, aproximadamente $20 \%{ }^{19,20}$, além de um intervalo muito curto da publicação do WHI e o último ano aqui estudado (2003).

Um fator que também poderia ter contribuído para o aumento do número de casos foi a melhora do rastreamento oportunístico na cidade de Goiânia ${ }^{21}$. Devido à inexistência de um programa nacional de rastreamento populacional, cada localidade vem desenvolvendo ações isoladas para que possam beneficiar determinadas populações (rastreamento oportunístico). Apesar de todas essas ações, a cobertura mamográfica no estado de Goiás foi de apenas $46 \%$ para mulheres com mais de 40 anos $^{22}$, taxa aquém dos $70 \%$ de cobertura mamográfica necessários para a redução da taxa de mortalidade pela neoplasia mamária ${ }^{23}$.

O carcinoma ductal invasor foi o tipo histológico mais frequente, seguido pelo carcinoma lobular invasor, achados semelhantes aos de outros estudos ${ }^{24,25}$.

Quanto ao estadiamento, os resultados apresentados no presente trabalho diferem daqueles observados em países desenvolvidos. Miller et al. ${ }^{24}$, em 2002, relataram que, nos Estados Unidos, os tumores localizados exclusivamente na mama foram mais frequentes, variando de 54 a $72 \%$ dos casos, seguido por tumores com extensão regional (comprometimento axilar), variando entre 23 e $38 \%$ dos casos. Para os tumores com metástases à distância ao diagnóstico, foram observadas taxas de 3 a $9 \%^{24}$. O presente estudo evidenciou que $45 \%$ dos casos de câncer de mama estiveram localizados exclusivamente na mama, $20 \%$ eram regionais e aproximadamente $10 \%$ dos casos foram diagnosticados com metástases à distância.

Este estudo temporal explica a diferença das taxas de casos avançados relatados por autores brasileiros na década de 1980 e início dos anos $1990^{7,8}$ em comparação aos estudos mais recentes ${ }^{9,10}$. Em decorrência do pequeno número de publicações a respeito do estadiamento, durante um longo período os pesquisadores brasileiros acreditaram que a taxa de doença avançada ainda estaria ao redor de $70 \%$, conforme mostrado pelos estudos baseados no diagnóstico de duas décadas atrás ${ }^{7}$. Os números registrados no último quinquênio são comparáveis aos trabalhos de outros autores brasileiros que publicaram suas experiências mais recentemente $\mathrm{e}^{9,10}$. Macchetti ${ }^{9}$, em um estudo com 106 mulheres que tiveram seu diagnóstico feito pelo Sistema Único de Saúde (SUS), observou que $76 \%$ dos casos foram detectados em estádios iniciais da doença e, destes, 6,6\% eram carcinoma in situ. Já Thuler e Mendonça ${ }^{10}$ avaliaram 7.458 pacientes provenientes de Centros de Alta Complexidade em Oncologia no período de 1990 a 1994, sendo que 43.442 casos eram provenientes de hospitais e serviços especializados de quimioterapia e radioterapia, entre 1995 e 2002. Os autores observaram uma significativa redução da taxa mediana de casos avançados, de 52 para $45 \%$, da primeira para a segunda fase do estudo ${ }^{10}$.

Um dado muito importante foi a observação crescente do número de casos de carcinoma in situ, que subiu de $0,2 \%$ no primeiro quinquênio para $6,2 \%$ no terceiro quinquênio estudado. A taxa de carcinoma in situ no terceiro quinquênio é muito parecida com aquela apresentada em São Carlos, no interior do Estado de São Paulo $(6,6 \%)^{9}$. Mesmo que a taxa de mulheres com diagnóstico de carcinoma in situ tenha aumentado no último quinquênio, ela está bem abaixo daquelas observadas nos países do leste europeu e dos Estados Unidos, que variam de 11 a $25 \%$, patamar alcançado em decorrência dos programas de rastreamento populacional ${ }^{26,27}$.

Deve ser notado que, no primeiro quinquênio, até 1995, o RCBP de Goiânia registrava os casos de comprometimento axilar como doença metastática. A partir daí foi introduzida a qualificação "regional", a explicação para a grande redução dos casos metastáticos, contrariamente ao aumento dos casos regionais (pacientes com linfonodos comprometidos). Ainda assim, mesmo depois desse ajuste, a taxa de pacientes com tumores metastáticos continuou diminuindo, mas houve um aumento paralelo dos casos regionais e, principalmente, dos casos de doença localizada na mama.

O presente estudo tem um ponto muito positivo. Diferentemente dos trabalhos conduzidos no Brasil e anteriormente citados, foram utilizados os dados do Registro de Base Populacional da cidade de Goiânia, o qual está em atividade ininterrupta por $20 \operatorname{anos}^{28} \mathrm{e}$ representa uma cidade de aproximadamente 1,3 milhões de habitantes, com uma taxa de crescimento populacional acima dos 2,5\% entre 2000 e $2007^{29}$. A maturidade na coleta das informações permite uma análise mais fidedigna e mostra claramente uma inversão dos casos tardios para diagnósticos nas fases iniciais da neoplasia maligna da mama. 
Assim, com o presente trabalho, observamos que houve um crescente aumento de casos novos de câncer de mama entre as moradoras de Goiânia durante os quinze anos analisados e que, comprovadamente, também houve melhora na codificação pelos médicos, que passaram a mostrar maior preocupação na confirmação através do exame histológico no último quinquênio. Por fim, o perfil diagnóstico do câncer de mama na cidade de Goiânia está mudando, e há um substancial aumento do diagnóstico de casos iniciais em detrimento de dos casos avançados.

\section{Referências}

1. Parkin DM, Bray F, Ferlay J, Pisani P. Global cancer statistics, 2002. CA Cancer J Clin. 2005;55(2):74-108.

2. Ministério da Saúde. Secretaria de Atenção à Saúde. Instituto Nacional do Câncer. Coordenação de Prevenção e Vigilância de Câncer. Estimativas 2008: incidência de câncer no Brasil. Rio de Janeiro: INCA; 2007.

3. Ministério da Saúde. Instituto Nacional de Câncer. Atlas de mortalidade por câncer no Brasil 1979-1999. Rio de Janeiro: INCA; 2002.

4. Lee JH, Yim SH, Won YJ, Jung KW, Son BH, Lee HD, et al. Population-based breast cancer statistics in Korea during 1993. 2002: incidence, mortality, and survival. J Korean Med Sci. 2007;22 Suppl:S11-6.

5. Freitas NMA, Freitas Júnior R, Curado MP, Martins E, Silva CMB, Moreira MMR, et al. Tendência da incidência e da mortalidade do câncer de mama em Goiânia: análise de 15 anos (1988-2002). Rev Bras Mastologia. 2006; 16(1):17-22.

6. Berry DA, Cronin KA, Plevritis SK, Fryback DG, Clarke L, Zelen $M$, et al. Effect of screening and adjuvant therapy on mortality from breast cancer. N Engl J Med. 2005;353(17):1784-92.

7. Silveira Júnior $L P$, Freitas Júnior $R$, Carneiro $A B$, Ribeiro LFJ, Queiroz GS. Fatores sócio-demográficos associados com o estadiamento das pacientes com câncer de mama. Rev Bras Ginecol Obstet. $1996 ; 18(5): 411-5$.

8. Caldeira JRF, Budin RMA. Aspectos epidemiológicos do câncer de mama em Jau-SP e a alta incidência de casos avançados em mulheres idosas. Rev Bras Cancerol. 1995;41(1):15-7.

9. Macchetti AH. Estadiamento do câncer de mama diagnosticado no sistema público de saúde de São Carlos. Medicina (Ribeirão Preto). 2007;40(3):394-402.

10. Thuler LCS, Mendonça GA. Estadiamento inicial dos casos de câncer de mama e colo do útero em mulheres brasileiras. Rev Bras Ginecol Obstet. 2005;27(1 1):656-60.

11. Jensen OM, Parkin DM, MacLennan R, Muir CS, Skeet RG. Cancer registration: principles and methods [IARC Scientific Publications, 95]. Lyon: IARC; 1991.

12. Ries LAG, Melbert D, Krapcho M, Stinchcomb DG, Howlader N, Horner M, et al., editors. SEER Cancer Statistics Review, 19752005. Bethesda: National Cancer Institute; 2005.

13. Freitas-Junior R, Freitas NM, Curado MP, Martins E, Moreira MA, Silva CM. Variations in breast cancer incidence per decade of life (Goiânia, GO, Brazil): 16-year analysis. Cancer Causes Control. 2008; 19(7):681-7.

14. Clagnan WS, Andrade JM, Carrara HHA, Tiezzi DG, Reis FJC, Marana HRC, et al. Idade como fator independente de prognóstico no câncer de mama. Rev Bras Ginecol Obstet. 2008;30(2):67-74.

15. Curado MP, Edwards B, Shin HR, Ferlay J, Heanue M, Boyle P, et al. Cancer incidence in five continents, Vol. IX [IARC Scientific Publications, 160]. Lyon: IARC; 2007.
16. Paulinelli RR, Freitas Júnior $R$, Curado MP, Souza AA. A situação do câncer de mama em Goiás, no Brasil e no mundo: tendências atuais para a incidência e a mortalidade. Rev Bras Saúde Matern Infant. 2003;3(1):17-24.

17. Katalinic A, Rawal R. Decline in breast cancer incidence after decrease in utilisation of hormone replacement therapy. Breast Cancer Res Treat. 2008;107(3):427-30.

18. Chlebowski RT, Kuller LH, Prentice RL, Stefanick ML, Manson JE, Gass $M$, et al. Breast cancer after use of estrogen plus progestin in postmenopausal women. N Engl J Med. 2009;360(6):573-87.

19. Machado RB, Fernandes CE, Maia E, Melo NR, Baracat EC. Hormone therapy following the Women's Health Initiative (WHI) study: perception of gynecologists in Brazil. Minerva Ginecol. 2005; 57(5):563-8.

20. Lazar F Jr, Costa-Paiva L, Morais SS, Pedro AO, Pinto-Neto AM. The attitude of gynecologists in São Paulo, Brazil 3 years after the Women's Health Initiative study. Maturitas. 2007;56(2):129-41.

21. Instituto Avon. Estratégias de articulação intersetorial: para a prevenção e detecção precoce do câncer de mama. São Paulo: Instituto Avon; 2006.

22. Instituto Brasileiro de Geografia e Estatística (IBGE). Diretoria de Pesquisas. PNAD 2003: acesso a utilização de serviços de saúde 2003. Rio de Janeiro: IBGE; 2005.

23. Smith RA, Cokkinides V, Brawley OW. Cancer screening in the United States, 2009: a review of current American Cancer Society guidelines and issues in cancer screening. CA Cancer J Clin. 2009;59(1):27-41

24. Miller BA, Hankey BF, Thomas TL. Impact of sociodemographic factors, hormone receptor status, and tumor grade on ethnic differences in tumor stage and size for breast cancer in US women. Am J Epidemiol. 2002;155(6):534-45.

25. Carey LA, Perou CM, Livasy CA, Dressler LG, Cowan D, Conway $\mathrm{K}$, et al. Race, breast cancer subtypes, and survival in the Carolina Breast Cancer Study. JAMA. 2006;295(21):2492-502.

26. Burstein HJ, Polyak K, Wong JS, Lester SC, Kaelin CM. Ductal carcinoma in situ of the breast. N Engl J Med. 2004;350(14):1430-41.

27. Van Steenbergen LN, Voogd AC, Roukema JA, Louwman WJ, Duijm $\mathrm{LE}$, Coebergh JWW, et al. Screening caused rising incidence rates of ductal carcinoma in situ of the breast. Breast Cancer Res Treat. $2009 ; 115(1): 181-3$

28. Moura L, Curado MP, Simões EJ, Cezário AC, Urdaneta M. Avaliação do registro de câncer de base populacional do município de Goiânia, Estado de Goiás, Brasil. Epidemiol Serv Saúde. 2006;15(4):7-17

29. Instituto Brasileiro de Geografia e Estatística (IBGE). Cidades. Goiânia. Pessoas residentes. Contagem da população 2007. [documento na Internet]. Rio de Janeiro; 2009 [citado $24 \mathrm{fev}$ 2009]. Disponível em: http://www.ibge.gov.br/cidadesat/ topwindow.htm. 